\title{
Prevalence and characteristics of TERT and TERC mutations in suspected genetic pulmonary fibrosis
}

\author{
Raphael Borie ${ }^{1,2}$, Laure Tabèze ${ }^{1,2}$, Gabriel Thabut ${ }^{2,3}$, Hilario Nunes ${ }^{4}$, \\ Vincent Cottin $\mathbb{1}^{5}$, Sylvain Marchand-Adam ${ }^{6}$, Grégoire Prevot ${ }^{7}$, Abdellatif Tazi $^{8}$, \\ Jacques Cadranel ${ }^{9}$, Herve $\mathrm{Mal}^{3}$, Lidwine Wemeau-Stervinou ${ }^{10}$, \\ Anne Bergeron Lafaurie ${ }^{8}$, Dominique Israel-Biet ${ }^{11}$, Clement Picard $^{12}$, \\ Martine Reynaud Gaubert ${ }^{13}$, Stephane Jouneau ${ }^{14}$, Jean-Marc Naccache ${ }^{9}$, \\ Julie Mankikian $^{6}$, Christelle Ménard ${ }^{15}$, Jean-François Cordier ${ }^{5}$, \\ Dominique Valeyre ${ }^{4}$, Marion Reocreux ${ }^{15}$, Bernard Grandchamp ${ }^{15}$ \\ Patrick Revy ${ }^{16}$, Caroline Kannengiesser ${ }^{15,17}$ and Bruno Crestani ${ }^{1,2,17}$
}

\begin{abstract}
Affiliations: ${ }^{1}$ APHP, Hôpital Bichat, Service de Pneumologie A, DHU FIRE, Centre de compétence des maladies pulmonaires rares, Paris, France. ${ }^{2}$ INSERM, Unité 1152; Université Paris Diderot, Paris, France. ${ }^{3}$ Service de Pneumologie B, APHP, Hôpital Bichat, Paris, France. ${ }^{4}$ APHP, Service de Pneumologie, Hôpital Avicenne, Bobigny, France. ${ }^{5}$ Service de Pneumologie, Centre national de référence des maladies pulmonaires rares, Hôpital Louis Pradel, Université Claude Bernard Lyon 1, Lyon, France. 'Service de Pneumologie, CHRU de Tours, Tours, France. ${ }^{7}$ Service de Pneumologie, Hôpital Larrey, Toulouse, France. ${ }^{8}$ APHP, Hôpital SaintLouis, Service de Pneumologie, Paris, France. ${ }^{9}$ APHP, Service de Pneumologie, Centre de compétence des maladies pulmonaires rares, Hôpital Tenon, Paris, France. ${ }^{10}$ Service de Pneumologie, Centre de compétence des maladies pulmonaires rares, CHRU de Lille, Lille, France. ${ }^{11}$ APHP, Hôpital Européen Georges Pompidou, Service de Pneumologie, Paris, France. ${ }^{12}$ Service de Pneumologie, Hôpital Foch, Suresnes, France. ${ }^{13}$ Service de Pneumologie, Hôpital Nord, Marseille, France. ${ }^{14}$ Service de Pneumologie, Centre de compétence des maladies pulmonaires rares, Hôpital Pontchaillou; IRSET UMR 1085, université de Rennes 1, Rennes, France. ${ }^{15}$ Departement de Génétique, APHP, Hôpital Bichat, Paris, France; Université Paris Diderot, Paris, France. ${ }^{16}$ INSERM UMR 1163, Laboratory of Genome Dynamics in the Immune System, Paris Descartes-Sorbonne Paris Cité University, Imagine Institute, Paris, France. ${ }^{17}$ Both authors contributed equally to this work. Additional collaborators are listed in the Acknowledgements section.
\end{abstract}

Correspondence: Bruno Crestani, Service de pneumologie A, Hôpital Bichat, 46 rue Henri Huchard, 75877 Paris Cedex 18, France. E-mail: bruno.crestanidaphp.fr

ABSTRACT Telomerase reverse transcriptase (TERT) or telomerase RNA (TERC) gene mutation is a major monogenic cause of pulmonary fibrosis. Sequencing of TERT/TERC genes is proposed to patients with familial pulmonary fibrosis. Little is known about the possible predictors of this mutation and its impact on prognosis.

We retrospectively analysed all the genetic diagnoses made between 2007-2014 in patients with pulmonary fibrosis. We evaluated the prevalence of TERT/TERC disease-associated variant (DAV), factors associated with a DAV, and the impact of the DAV on survival.

237 patients with pulmonary fibrosis (153 with familial pulmonary fibrosis, 84 with telomere syndrome features without familial pulmonary fibrosis) were tested for TERT/TERC DAV. DAV was diagnosed in 40 patients (16.8\%), including five with non-idiopathic interstitial pneumonia. Prevalence of TERT/TERC DAV did not significantly differ between patients with familial pulmonary fibrosis or with only telomere syndrome features (18.2\% versus $16.4 \%$ ). Young age, red blood cell macrocytosis, and low platelet count were associated with the presence of DAV; the probability of DAV was increased for patients 40-60 years. Transplant-free survival was lower with than without TERT/TERC DAV (4.2 versus 7.2 years; $\mathrm{p}=0.046$ ).

TERT/TERC DAV were associated with specific clinical and biological features and reduced transplantfree survival.

@ERSpublications

Pulmonary fibrosis patients with TERT/TERC disease-associated variants show reduced transplant-free survival http://ow.ly/EmYs304atGl 


\section{Introduction}

The genetic background of interstitial lung disease (ILD) features the prevalence of idiopathic pulmonary fibrosis (IPF), which is $0.02 \%$ in the general population; however, $2-20 \%$ of IPF patients are from families with ILD [1,2]. Genetic studies of familial forms of IPF led to the discovery of mutations within telomerase reverse transcriptase (TERT) and telomerase RNA (TERC), both required for normal telomerase activity [3, 4]. Heterozygous mutations in TERT/TERC are detected in $\sim 15 \%$ of patients with familial ILD but rarely in sporadic IPF $(<3 \%)$ [3-5]. Mutations in TERT/TERC are also associated with extra-pulmonary abnormalities, assessed as telomere syndrome, involving skin, liver and haematological abnormalities [6-8]. The phenotype may be heterogeneous, even in patients with the same mutation [9]. In addition, a polymorphism in MUC5B is strongly associated with IPF, whether sporadic or familial $[5,10,11]$.

International guidelines for IPF in 2011 did not recommended genetic testing in the state of understanding, but international guidelines for idiopathic interstitial pneumonia in 2013 and French practical guidelines suggest searching for genetic abnormalities in patients with familial pulmonary fibrosis (FPF) [12-14]. However, TERT/TERC mutation testing indications need to be clarified, as does the clinical impact of these mutations. The aim of this study was to evaluate the prevalence of TERT/TERC mutation in a national cohort of ILD with suspected TERT/TERC mutation in France, to search for factors predicting these mutations and to evaluate their impact on survival.

\section{Methods}

\section{Patients}

This was a retrospective, observational, non-interventional study. All patients referred for TERT/TERC sequencing in the genetic laboratory of Bichat Hospital (Paris, France) between 2007 and January 2014 were screened for the study. This laboratory is the reference facility in France for TERT/TERC mutation testing and belongs to a network including adult and paediatric centres with a special interest in rare lung diseases. For this study, we included all probands with ILD and suspected TERT/TERC mutation. All patients were referred by a pulmonologist for a genetic diagnosis of the ILD. Patients referred for dyskeratosis congenita were not included; however, when reviewing the clinical chart, one patient fulfilled the dyskeratosis congenita criteria and was not excluded [15]. Patients with hepato-pulmonary syndrome were not included in the study.

The clinical charts for patients were reviewed and data were collected by use of a standardised and anonymous collection form. Personal and family history of pulmonary diseases (particularly ILD), haematological disorders (including abnormal blood count), liver abnormalities (repeated unexplained elevated liver enzyme levels or liver disease) and personal cutaneous abnormalities (hair greying before the age of 30, skin hyperpigmentation, oral leukoplakia, and nail dystrophy) were specifically assessed. The results of standard biological tests were collected at the diagnosis of ILD. Red blood cell macrocytosis was defined by mean corpuscular volume $>100 \mathrm{fL}$ and thrombocytopenia by platelet count $<150 \mathrm{~g} \cdot \mathrm{L}^{-1}$ according to the World Health Organization definition [16]. In this study, personal or familial hepatic or haematological diseases and personal cutaneous signs were considered extra-pulmonary involvement.

Lung diagnosis was based on a multidisciplinary team discussion including clinical data, lung high-resolution computed tomography (HRCT) and pathological evaluation, according to the 2011 official American Thoracic Society (ATS)/European Respiratory Society (ERS)/Japanese Respiratory Society (JRS)/ Latin American Thoracic Association (ALAT) statement for IPF [14, 17] and the revised classification of idiopathic interstitial pneumonia [13]. Patient with possible usual interstitial pneumonia pattern on computed tomography scan without available histology who were given a working diagnosis of IPF after multidisciplinary discussion were classified as such in this study. Professional or domestic exposure to inhaled fibrogenic particles (such as asbestos) or antigens possibly associated with chronic hypersensitivity pneumonitis (such as chronic exposure to birds or moulds) (see online data supplement) was systematically considered, as was the use of drugs associated with pulmonary fibrosis according to publicly available databases (www.pneumotox.com). Tobacco smoke exposure was quantified. The results of lung function tests obtained at the diagnosis of ILD were collected. Death and lung transplantation data were collected up to September 1, 2015, except for cases lost to follow-up.

Editorial comment in: Eur Respir J 2016; 48: 1556-1558

This article has supplementary material available from erj.ersjournals.com

Received: Dec 142015 | Accepted after revision: Sept 032016 | First published online: Nov 112016

Support statement: This work was supported by the Chancellerie des Universités de Paris (legs Poix) and by a grant "FPI-SPC" from Université Sorbonne Paris Cité.

Conflict of interest: Disclosures can be found alongside this article at erj.ersjournals.com 
According to national rules in France, all patients or relatives gave a signed consent for germline genetic analysis. The Institutional Review Board of the French Society for Respiratory Medicine (Société de Pneumologie de Langue Française) approved this study (CEPRO 2015-020).

\section{Genetic analysis}

Methods for sequencing are presented in the online data supplement. Patients with TERT and TERC rare variants were classified according to the American College of Genetics and Genomics guidelines and European Society for Human Genetics recommendations [18, 19]. Briefly, there are five head categories of variants: pathogenic, likely pathogenic, uncertain significance (VUS), likely benign and benign. The following supporting, moderate or strong criteria were used when available: nature of the variant (very strong to supporting), previous report (strong), population data (moderate), segregation data (strong to supporting), telomere length as functional data (supporting), computational and predicitive data such as polyphen or combined annotation-dependent depletion (CADD) scores (supporting) and specific phenotype (supporting). The combination of one moderate criteria and four or more supporting criteria for pathogenicity leads to a conclusion of likely pathogenic [18]. Within the group of VUS, variants with one moderate criteria and three supporting criteria were considered as VUS possibly disease associated (VUSD). All other VUS were considered as VUS possibly benign (VUSB). Variants classified as pathogenic, likely pathogenic and VUSD were retained as disease-associated variant (DAV).

\section{Statistical analysis}

Continuous variables are expressed as median (range) and were compared by t-test. Categorical variables are expressed as $\mathrm{n}(\%)$ and were compared by Chi-squared test. Comparison of patients with TERT/TERC mutation involved non-parametric tests because of the small sample sizes.

Because a substantial number of patients underwent lung transplantation, traditional survival analyses in which patients undergoing lung transplantation would be censored at the time of transplant would provide biased estimates (informative censoring). Therefore, we used time to death or transplant (transplant-free survival) as our primary end-point. The starting point was the date of ILD diagnosis. Univariate and multivariate Cox models were used to assess the impact of baseline covariates on transplant-free survival with hazard ratios. The correlation of observations within centres was accounted for by use of a grouped Jackknife estimator [20].

Factors associated with TERT/TERC DAV were tested by comparing carriers and non-carriers and by fitting univariate and multivariate logistic regression models. The centre-effect was accounted for by using generalised estimating equation models. The coxph function (survival package) with the cluster option was used to compute Cox models, and the gee function (gee package) was used to compute logistic models. All tests were two-sided, with $\mathrm{p}=0.05$ indicating statistical significance. All analyses involved use of $\mathrm{R}$ software (version 2.15.2; www.r-project.org).

\section{Results}

\section{Patients}

On January 1, 2014, DNA from 237 probands with ILD (153 with FPF and 84 non-FPF with extra-pulmonary involvement) was tested for suspected TERT/TERC mutation, including 11 patients previously reported [21-23] (figure 1). The mean age was 61.3 years (21.4-92.6), with a male predominance $(71.4 \%)$ (table 1$)$. The final pulmonary diagnosis was IPF $(n=97)$, probable, possible IPF or working diagnosis of IPF $(n=73)$, suspected idiopathic nonspecific interstitial pneumonia $(n=12)$, chronic hypersensitivity pneumonitis $(n=13)$, drug-induced pneumonitis $(n=4)$, desquamative interstitial pneumonia $(n=3)$, respiratory bronchiolitis with ILD $(n=1)$, collagen vascular disease associated ILD ( $n=17$ : rheumatoid arthritis, $n=6$; Sjögren's syndrome, $n=5$; undifferentiated connective tissue disease, $n=2$; systemic sclerosis, $\mathrm{n}=1$; fibrosis with antineutrophil cytoplasmic autoantibody-associated vasculitis, $\mathrm{n}=2$; systemic lupus erythematosus, $n=1)$, pneumoconiosis $(n=3)$, idiopathic pleuroparenchymal fibroelastosis $(n=2)$, cryptogenic organising pneumonia $(n=1)$, pulmonary fibrosis associated with alveolar proteinosis $(\mathrm{n}=1)$ and nonclassifiable fibrosis $(\mathrm{n}=10)$. Thus 195 patients with idiopathic ILD, including 170 with IPF and 42 with secondary ILD, were included.

Details on the hepatic and haematological diseases and cutaneous signs are in the online data supplement.

\section{Carriers of TERT/TERC mutation}

Genetics

We identified 31 (13.0\%) probands with pathogenic or likely pathogenic TERT/TERC variants, 26 (10.9\%) within TERT and five (2.1\%) within TERC (tables 2 and E1). Nine variants were classified as VUSD and 11 as VUSB. As the group of patient carriers of pathogenic or likely pathogenic TERT/TERC variants did 


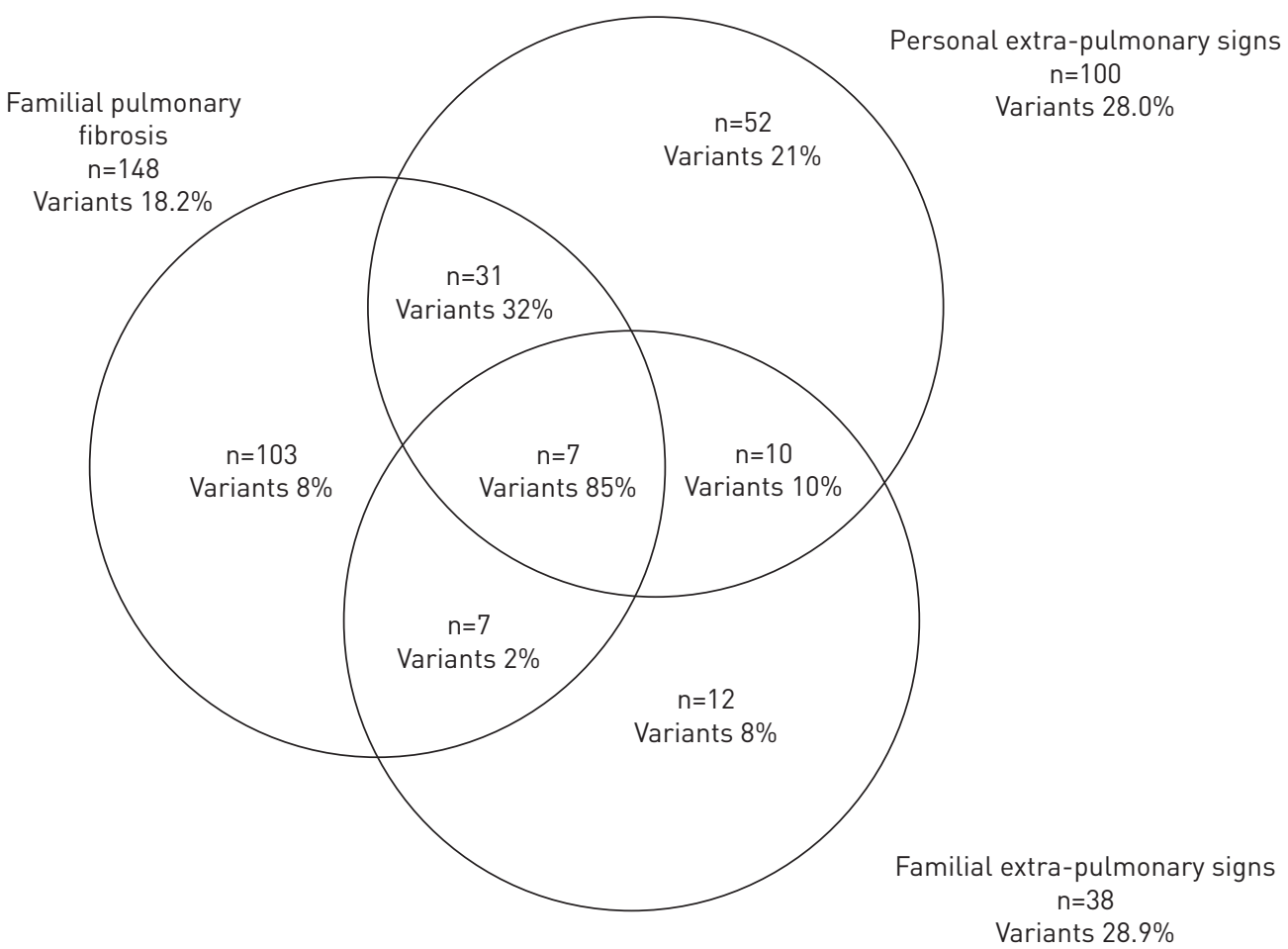

FIGURE 1 Probands included in the study by what led to suspicion of a mutation and percentage of patient carriers of disease-associated variant in each group.

not differ significantly from the group of patient carriers of VUSD, both groups were gathered, called patients with TERT/TERC DAV and compared with the group without any variant. Carriers of VUSB within TERT or TERC were not included in further analysis. Four ultra-rare variants were found several times in three, two, two and two probands, respectively (table E1). As far as we know, none of them were relatives as they did not share the same name nor did they come from the same area.

Figure 1 displays the proportion of patients with TERT/TERC DAV by presence of FPF and/or extra-pulmonary involvement. Among FPF patients, the proportion with mutation was $18.2 \%$. The highest proportion with mutation was for those with FPF and extra-pulmonary signs (16 (42.1\%) out of 38).

Patients with TERT/TERC DAV were genotyped for the MUC5B rs35705950 polymorphism. The frequency of the minor-allele (at-risk) $M U C 5 B$ polymorphism was 19 (25\%) out of 74 for those with TERT/TERC DAV and was greater than for controls $\left(\mathrm{n}=1229,10.8 \% ; \mathrm{p}=8 \times 10^{-66}\right)$ but lower than for a French IPF cohort $(\mathrm{n}=142,41.9 \% ; \mathrm{p}=0.02)$ [5].

Mean length of telomere was significantly reduced in TERC DAV carriers when compared to TERT DAV carriers or to controls at $4.7 \mathrm{~kb}, 7.1 \mathrm{~kb}$ and $7.6 \mathrm{~kb}$ respectively $(\mathrm{p}=0.01$ and $\mathrm{p}=0.002)$ (figure E1a). When compared with the normal values, all patients with TERC DAV and 11 patients with pathogenic or likely pathogenic TERT variants showed reduced telomere length. Within carriers of TERT DAV, patients self-reported to be from the second generation affected, showed shorter telomere than patients from the first generation, $(\mathrm{p}=0.02)$ (figure $\mathrm{E} 1 \mathrm{~b})$.

\section{Pulmonary involvement}

The mean (range) age of patient carriers of TERT/TERC DAV was 57 (36-79) years (table 2). Most (55.2\%) were smokers, and $42.1 \%$ had chronic exposure to potentially pneumotoxic dust (asbestos, metals, wood, birds, moulds, etc.); $73.6 \%$ were smokers or had chronic pneumotoxic exposure. The final pulmonary diagnosis was IPF $(n=24,60.0 \%)$, nonclassifiable fibrotic ILD $(n=7,17.5 \%)$, chronic hypersensitivity pneumonitis $(n=3,7.5 \%)$, suspected idiopathic nonspecific interstitial pneumonia $(n=4$, $10.0 \%)$, rheumatoid arthritis-associated ILD $(n=1,2.5 \%)$ and drug-induced ILD $(n=1,2.3 \%)$. Thus, five patients (12.5\%) showed a secondary form of ILD (hypersensitivity pneumonitis, connective tissue disease or drug induced). 
TABLE 1 Main characteristics of the 237 patients

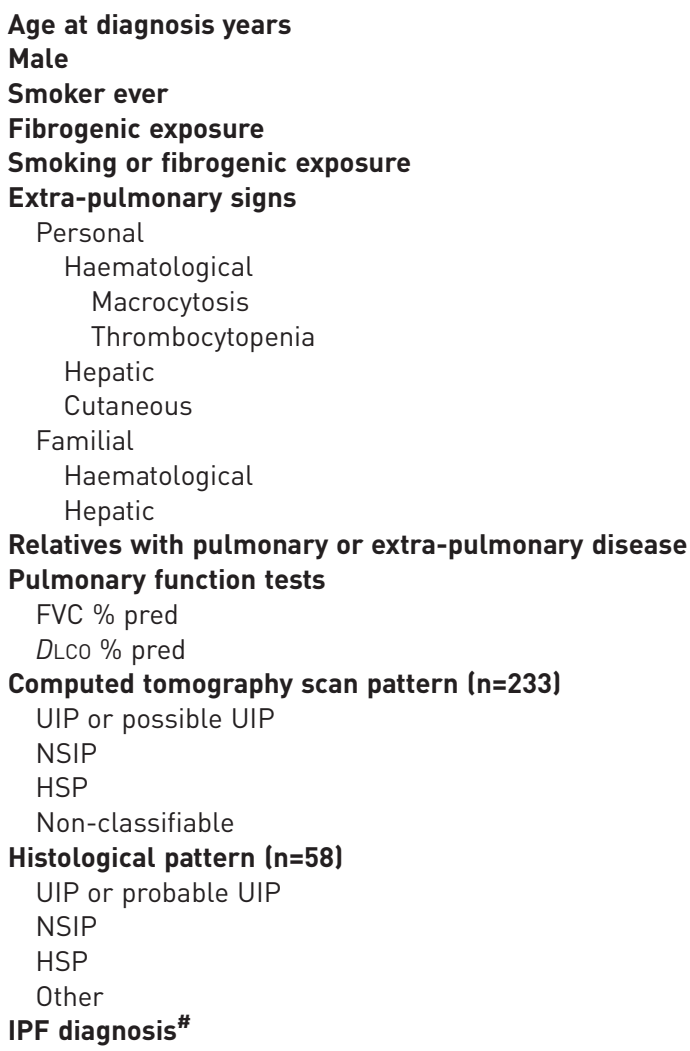

$61.3(21.4-92.6)$

$170(71.7)$

$135(56.9)$

$75(31.6)$

$170(71.7)$

$135(56.9)$

$117(49.3)$

$75(31.6)$

$42(17.7)$

57 (24.0)

39 (16.4)

27 (11.3)

40 (16.8)

19 (8.0)

24 (10.1)

$1.4(0-7)$

74 (25-149)

47 (13-87)

127 (53.5)

42 (17.7)

5 (2.1)

43(18.1)

$38(64.9)$

$4(6.8)$

$3(4.4)$

13 (22.4)

170 (71.7)

Data are presented as mean (range) or $\mathrm{n}(\%)$. Forced vital capacity (FVC) and diffusing capacity of the lung for carbon monoxide (DLCO) are expressed as \% predicted value. UIP: usual interstitial pneumonia; NSIP: nonspecific interstitial pneumonia; HSP: hypersensitivity pneumonitis; IPF: idiopathic pulmonary fibrosis.

\#: IPF or probable IPF or possible IPF diagnosis based on computed tomography scan and histology.

Patients with possible UIP on computed tomography scan without histology but with a working diagnosis of

IPF after multidisciplinary discussion are classified as IPF.

\section{Extra-pulmonary involvement}

Personal haematological abnormalities were of two kinds. A total of 12 patients presented only abnormal blood cell count without identified bone marrow disease: low platelet count $(n=6,15.0 \%)$, red blood cell macrocytosis $(n=3,7.5 \%)$, or both $(n=3,7.5 \%)$; in 11 patients, bone marrow diseases were identified by bone-marrow aspirate or bone-marrow biopsy: myelodysplasia $(n=5,12.5 \%)$, bone-marrow failure $(n=2$, $5.0 \%)$, myelofibrosis $(\mathrm{n}=1,2.5 \%)$ and acute myeloid leukemia $(\mathrm{n}=1,2.5 \%)$. Myelodysplasia developed in two patients with thrombocytopenia during follow-up; one patient had acute myeloid leukemia and one myelodysplasia without previous cytopenia. Furthermore, V617F mutation of JAK2 was evidenced in two patients with myelodysplasia.

In all, 13 patients showed liver disorders: repeated unexplained elevated liver enzymes $(\mathrm{n}=5,12.5 \%)$, non-alcoholic steato-hepatitis $(\mathrm{n}=4,10.0 \%)$, idiopathic liver cirrhosis $(\mathrm{n}=3,7.5 \%)$, and focal nodular hyperplasia $(\mathrm{n}=1,2.5 \%)$. Hepatopulmonary syndrome was not explicitly evaluated and no cases were documented.

Overall, 11 patients showed skin abnormalities: skin hyperpigmentation $(n=5,12.5 \%)$, premature hair greying $(n=5,12.5 \%)$, oral leukoplakia $(n=1,2.3 \%)$, and nail dystrophy $(n=1,2.3 \%)$. The total is greater than 11 because patients could present several signs. Only one patient with skin hyperpigmentation, oral leukoplakia and nail dystrophy fulfilled the dyskeratosis congenita criteria.

\section{Predictors of TERT/TERC mutation}

We did not find any significant difference between carriers of TERT or TERC DAV in age, sex ratio, tobacco smoking, fibrogenic exposure, extra-pulmonary signs, pulmonary function test alterations, histological or HRCT pattern, or final diagnosis. We further compared patients with and without TERT/TERC DAV. 
TABLE 2 Main characteristics of patients by presence of telomerase reverse transcriptase (TERT)/telomerase RNA (TERC) gene variants

\begin{tabular}{|c|c|c|c|c|}
\hline $\begin{array}{l}\text { Pathogenic or likely } \\
\text { pathogenic }\end{array}$ & TERT/TERC VUSD & TERT/TERC VUSB & $\begin{array}{c}\text { Absence of } \\
\text { TERT/TERC variant }\end{array}$ & $\begin{array}{c}\text { Univariate } \\
\text { analysis p-value }\end{array}$ \\
\hline
\end{tabular}

\begin{tabular}{|c|c|c|c|c|c|}
\hline Number & $31(13.0)$ & 9 (3.7) & $11(4.6)$ & $186(78.1)$ & \\
\hline Age years & 59.3 (39.3-82.8) & $54.4(41.5-69.5)$ & $57.9(30.2-76.8)$ & $63.0(24.7-92.6)$ & 0.02 \\
\hline Male & $22(70.9)$ & $6(66.6)$ & 9 (81.8) & 134 (71.5) & 0.64 \\
\hline Smoker ever & 17 (54.8) & $4(44.4)$ & $4(36.3)$ & $110(64.7)$ & 0.44 \\
\hline Smoking or fibrogenic exposure & $22(70.9)$ & $6(66.6)$ & 6 (54.5) & 135 (72.5) & 0.74 \\
\hline Familial pulmonary fibrosis & $23(74.2)$ & 3 (33.3) & $5(41.6)$ & $118(63.4)$ & 0.85 \\
\hline Extra-pulmonary signs & $24(77.4)$ & $7(77.7)$ & $8(72.7)$ & 90 (48.3) & 0.0008 \\
\hline Macrocytosis & $14(45.1)$ & $1(11.1)$ & $5(45.4)$ & $21(11.2)$ & $<0.001$ \\
\hline Thrombocytopenia & $17(54.8)$ & $3(33.3)$ & $4(36.3)$ & 35 (18.8) & $<0.001$ \\
\hline Hepatic & $9(29.0)$ & $4(44.4)$ & $4(36.3)$ & $22(12.2)$ & 0.001 \\
\hline Cutaneous & $10(32.2)$ & $1(11.1)$ & $2(18.1)$ & $15(8.4)$ & $<0.001$ \\
\hline Familial & $9(29.0)$ & $2(22.2)$ & $3(27.2)$ & $27(14.5)$ & 0.04 \\
\hline Haematological & $5(16.1)$ & $0(0)$ & $1(9.0)$ & $13(7.1)$ & 0.24 \\
\hline \multicolumn{6}{|l|}{ Pulmonary function tests } \\
\hline FVC & $75(25-91)$ & 63 (43-99) & $40(19-77)$ & $77(28-67)$ & 0.18 \\
\hline$D\llcorner c o$ & $52(13-53)$ & $46(24-80)$ & $62(35-93)$ & $47(14-62)$ & 0.85 \\
\hline \multicolumn{6}{|l|}{ Computed tomography pattern } \\
\hline UIP or possible UIP & $18(58.0)$ & 5 (55.5) & $7(63.6)$ & $98(52.6)$ & 0.57 \\
\hline IPF diagnosis & $18(58.0)$ & $6(66.6)$ & 8 (72.7) & $138(74.1)$ & 0.01 \\
\hline \multicolumn{6}{|c|}{$\begin{array}{l}\text { Data are presented as mean (range) or } \mathrm{n}(\%) \text {, unless otherwise indicated. Statistical analysis included pathogenic, likely pathogenic variants } \\
\text { and uncertain significance variants (VUS) possibly disease associated (VUSD) versus absence of variant. FVC: forced vital capacity; DLco: } \\
\text { diffusing capacity of the lung for carbon monoxide; UIP: usual interstitial pneumonia; IPF: idiopathic pulmonary fibrosis; VUSB: VUS possibly } \\
\text { benign. }\end{array}$} \\
\hline
\end{tabular}

As compared to the patients without TERT/TERC DAV, those with a DAV were younger, less frequently had a diagnosis of IPF and more frequently had extra-pulmonary signs (table 2).

Plots based on smoothing splines supported a non-linear association between age at diagnosis and the probability of TERT/TERC DAV; probability of being a carrier was greater for patients 40-60 years old than younger patients ( $<40$ years) and older patients ( $>60$ years); a threshold could not be easily defined (figure 2). HRCT pattern, presence of secondary ILD and severity of ILD assessed by pulmonary function tests were not associated with the mutation.

On multivariate analysis cutaneous abnormalities $(p=0.006)$, low platelet count $(p=0.05)$, familial liver disorders $(\mathrm{p}=0.01)$ and number of relatives with lung disease $(\mathrm{p}=0.004)$ remained significantly associated with TERT/TERC DAV. Age at diagnosis was not significantly associated with TERT/TERC variant in this model $(\mathrm{p}=0.21)$. A model including these 5 variables showed fair discrimination, with an area under receiver operating characteristic curve (AUC) of 0.74 (95\% CI: 0.64-0.85). Accordingly, a score including all these 5 variables had no clear advantage as compared with the variables alone. Among the 5 variables in the final model, low platelet count had the highest discriminative ability (AUC of 0.67 (0.57-0.77)).

If we limited the analysis to the subgroup of patients with FPF, the factors young age $(p=0.07)$, increased number of relatives with lung disease $(\mathrm{p}=0.06)$, life-long non-smoking $(\mathrm{p}=0.02)$ and presence of personal or familial extra-pulmonary signs $(\mathrm{p}<0.0001)$ were associated with the presence of a TERT/TERC DAV.

\section{Survival}

Overall and transplant-free survival

Median (range) follow-up time for the whole population $(n=226)$ was 3.7 years $(0.1-18.4$ years). During follow-up, 69 (30.5\%) patients died, 27 (11.9.0\%) underwent lung transplantation and the 130 remaining patients $(57.5 \%)$ were still alive at the end of the study. 


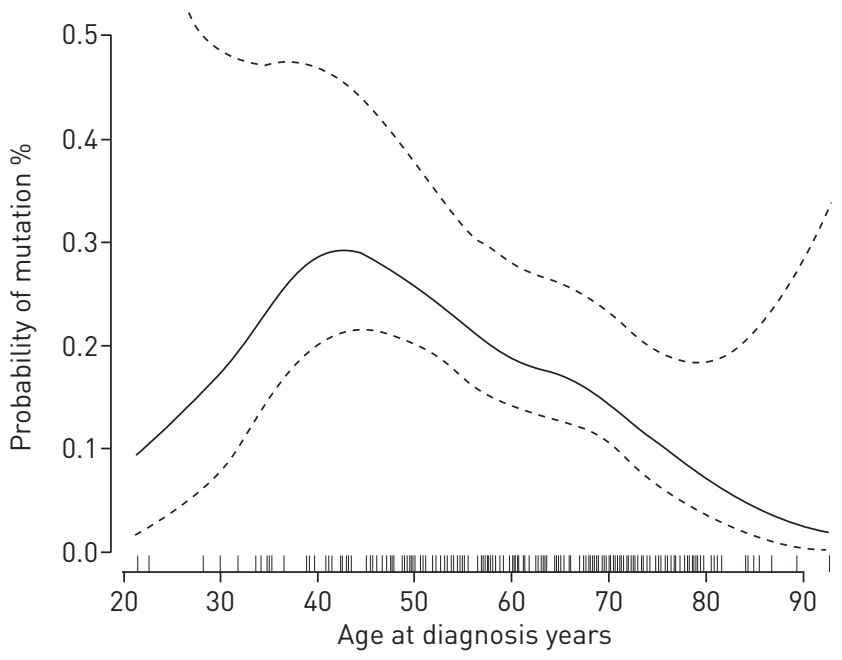

FIGURE 2 Association between age at diagnosis and the probability of telomerase reverse transcriptase (TERT)/telomerase RNA (TERC) gene disease-associated variant (DAV). Plots based on smoothing splines. Younger age was significantly associated with the presence of a DAV without linear regression between the probability of a DAV and the age. The smoothing splines support an increased probability of a DAV in the 40-60-year-old patient.

Median (95\% CI) transplant-free survival for the whole cohort was 6.3 years (5.1-8.1 years). Transplant-free survival was $73.5 \%(67.4-80.0 \%), 58.99 \%(51.7-67.1 \%)$ and $26.6 \%(17.1-41.3 \%)$ at 3, 5 and 10 years, respectively. The median follow-up did not significantly differ between carriers and non-carriers of TERT/ TERC DAV (3.3 years (0.4-13.8 years) versus 3.4 years $(0.1-18.4$ years); $\mathrm{p}=0.90)$. Transplant-free survival was not different according the presence of the MUC5B rs35705950 polymorphism in the TERT/TERC DAV carriers. Median transplant-free survival was lower for carriers than non-carriers (4.2 (2.9-infinity) versus $7.2(6.0-9.4)$ years; log-rank test $\mathrm{p}=0.046)$ (figure 3 ). Transplantation was more frequent for patients with than without TERT/TERC DAV (11 (30.2\%) versus 16 (9.2\%); $\mathrm{p}=0.002)$. Age at transplantation did not differ between carriers and non-carriers ( 52.5 versus 54.4 years; $\mathrm{p}=0.6$ ).

Factors associated with transplant-free survival

The association between variables measured at baseline and transplant-free survival is given in table 3 . Presence of TERT/TERC DAV, male sex, decreased force vital capacity or diffusing capacity of the lungs for carbon monoxide as well as red blood cell macrocytosis, and thrombocytopenia were significantly associated with reduced transplant-free survival. Transplant-free survival was decreased with presence of a

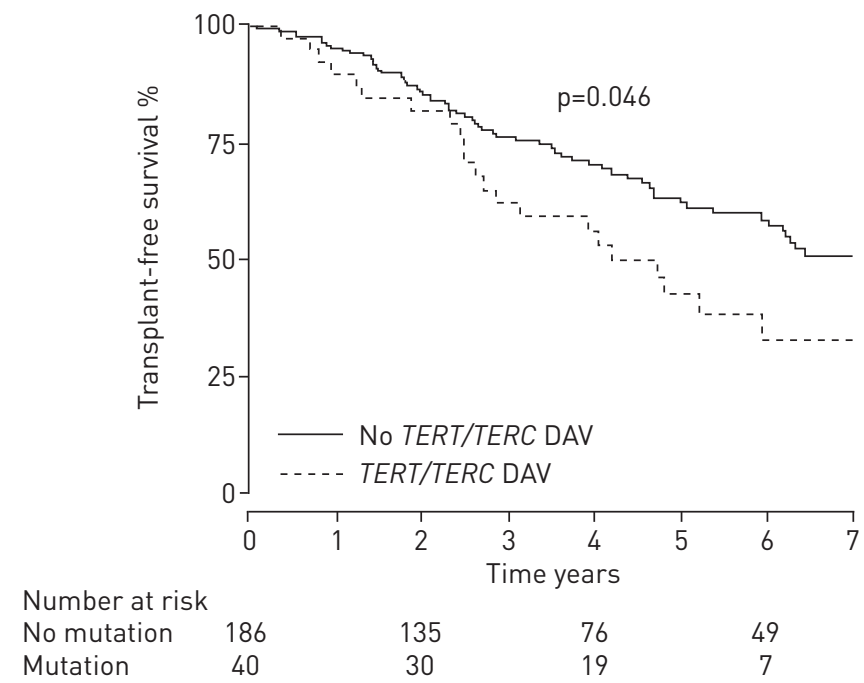

FIGURE 3 Transplant-free for patient carriers and non-carriers of telomerase reverse transcriptase (TERT)/ telomerase RNA (TERC) gene disease-associated variant (DAV). Median transplant-free survival was reduced for DAV carriers ( $p=0.046)$. 
TABLE 3 Factors associated with transplant-free survival

\begin{tabular}{|c|c|c|c|c|}
\hline & \multicolumn{2}{|c|}{ Univariate analysis } & \multicolumn{2}{|c|}{ Multivariate analysis } \\
\hline & $\operatorname{HR}(95 \% \mathrm{CI})$ & p-value & $\operatorname{HR}(95 \% \mathrm{CI})$ & p-value \\
\hline Age at diagnosis by 5 years & $1.07(0.97-1.17)$ & 0.15 & $1.18(1.06-1.31)$ & 0.002 \\
\hline TERT/TERC DAV carrier & $1.63(1.08-2.47)$ & 0.02 & $1.92(1.15-4.13)$ & 0.003 \\
\hline Male & $2.00(1.21-3.22)$ & 0.006 & $2.08(1.42-3.03)$ & $<0.001$ \\
\hline Smoker ever & $1.16(0.8-1.69)$ & 0.44 & & \\
\hline \multicolumn{5}{|l|}{ Extra-pulmonary signs } \\
\hline \multicolumn{5}{|l|}{ Personal } \\
\hline Haematological & $1.67(1.24-2.24)$ & 0.001 & & \\
\hline Macrocytosis & $2.45(1.86-3.22)$ & $<0.001$ & & \\
\hline Thrombocytopenia & $2.00(1.54-2.59)$ & 0.006 & & \\
\hline Hepatic & $1.38(0.98-1.94)$ & 0.06 & & \\
\hline Cutaneous & $1.56(1.13-2.15)$ & 0.007 & & \\
\hline \multicolumn{5}{|l|}{ Familial } \\
\hline Haematological & $0.70(0.3-1.63)$ & 0.41 & & \\
\hline Hepatic & $0.72(0.44-1.16)$ & 0.17 & & \\
\hline $\begin{array}{l}\text { Number of relatives with pulmonary } \\
\text { or extra-pulmonary signs }\end{array}$ & $0.88(0.74-1.05)$ & 0.17 & & \\
\hline \multicolumn{5}{|l|}{ Pulmonary function } \\
\hline FVC by 0.1 units & $0.84(0.75-0.93)$ & 0.001 & $0.81(0.72-0.92)$ & $<0.001$ \\
\hline DLco by 0.1 units & $0.69(0.58-0.81)$ & $<0.001$ & & \\
\hline IPF final diagnosis & $1.34(0.9-1.98)$ & 0.15 & & \\
\hline
\end{tabular}

TERT: telomerase reverse transcriptase; TERC: telomerase RNA; DAV: disease-associated variant; FVC: forced vital capacity; $D\llcorner C 0$ : diffusing capacity of the lung for carbon monoxide; IPF: idiopathic pulmonary fibrosis.

DAV in a multivariable model including age, sex and forced vital capacity and controlling for centre (HR 1.92 (95\% CI 1.15-4.13); $\mathrm{p}=0.003$ ). Similar results were found when the analysis was limited to patients with a diagnosis of IPF (HR 2.36 (1.35-4.13); $\mathrm{p}=0.003)$. Results were consistent whatever the computed tomography scan probability of usual interstitial pneumonia.

\section{Causes of death}

Among the 69 deaths, 36 (52.1\%) were considered related to fibrosis (end-stage pulmonary fibrosis, acute exacerbation or pulmonary hypertension) and 33 (47.8\%) not related (cirrhosis, infection, refractory pancytopenia, cardiac failure, stroke, pulmonary embolism or unknown). For patients presenting refractory pancytopenia, five TERT/TERC DAV carriers died versus two non-carriers $(\mathrm{p}=0.01)$. The frequency of death due to end-stage fibrosis was similar in the two groups (five out of 17 versus 31 out of $62 ; \mathrm{p}=0.13$ ).

\section{Discussion}

This series is a national cohort of ILD of suspected monogenic cause with systematic sequencing of TERT/ TERC and many participating centres $(\mathrm{n}=31)$. In patients with suspected TERT/TERC DAV, this series shows that 1) extra-pulmonary involvement and age at diagnosis predicted the presence of TERT/TERC DAV, 2) TERT/TERC DAV could be associated with different pulmonary phenotypes including non-idiopathic ILD, and 3) the presence of a TERT/TERC DAV was associated with reduced transplant-free survival.

We included all patients with ILD and suspected TERT/TERC mutation. The ATS/ERS/JRS/ALAT statement on idiopathic interstitial pneumonia suggests performing TERT/TERC sequencing for cases of familial ILD and does not consider extra-pulmonary signs [13], whereas national recommendations for clinical practice in France suggest also performing TERT/TERC sequencing in patients with extra-pulmonary signs $[14,24]$. Our results support broader recommendations because the mutation rate was similar for patients with FPF (18.2\%) and patients without familial occurrence of ILD but with extra-pulmonary involvement suggestive of a telomere syndrome (16.4\%). This rate of detection is similar to that observed in previous studies of FPF $[3,4]$.

Overall, 37.5\% of our patients with TERT/TERC DAV had red blood cell macrocytosis, 50.0\% thrombocytopenia and $32.5 \%$ liver abnormalities, all predictive of TERT/TERC DAV. TERT/TERC DAV carriers in previous cohorts showed extra-pulmonary signs $[9,25,26]$. For instance, bone-marrow failure associated with pulmonary fibrosis in the same individual or family was found highly predictive of TERT/ TERC mutation [27]. TERT/TERC mutation has been associated with cryptogenic cirrhosis, 
hepato-pulmonary syndrome, dyskeratosis congenita and premature hair greying [7, 28]. The classic triad of dyskeratosis congenita is dysplastic nail, skin hyperpigmentation and oral leukoplakia [15]. After reviewing of the chart, one patient fulfilled the criteria and was, as suspected, carrier of a TERT DAV. However, as far as we know, the rate of thrombocytopenia or macrocytosis in patients with ILD and TERT/TERC mutations was not previously reported. The shortest telomere length was found in subjects with TERC DAV, suggesting an increased penetrance and a higher risk or telomere syndrome for these patients [8]. The lack of clinical difference between TERT and TERC DAV carriers in this study could be related to the small number of TERC DAV carriers included, and needs to be further evaluated.

In the current series, five out of 40 patients with DAV were considered to present non-idiopathic ILD compared with 35 out of 186 in the non-carrier group $(p=0.34)$. Non-IPF ILD may have a genetic background. Indeed, in a series of 114 patients with a diagnosis of chronic hypersensitivity pneumonitis, $17.5 \%$ had a family member with pulmonary fibrosis, an argument that favours a genetic continuum between non-idiopathic and idiopathic ILD [29]. Moreover, in a series of 29 patients with surgical specimen of ILD, DIAZ DE LEON et al. [9] reported two cases of chronic hypersensitivity pneumonitis. Furthermore, we and others recently reported RTEL1, PARN and TERT mutations in patients with non-idiopathic ILD (such as connective tissue disease-associated ILD, pneumoconiosis, chronic hypersensitivity pneumonitis) in the context of $\operatorname{FPF}[23,30,31]$. This series evidence that non-IPF ILD and non-idiopathic ILD should indicate the need for genetic testing in cases of FPF or telomere syndrome.

Almost two-thirds of TERT/TERC DAV carriers in this series reported smoking or fibrogenic exposure. In a US cohort of 134 patients from 21 families with TERT mutation, almost all patients with ILD (96\%) were smokers or declared a pneumotoxic exposure [9]. TERT/TERC mutation has also been reported in viral or alcoholic cirrhosis series [32]. Furthermore, patients with TERT/TERC mutation can present severe haematological complications after lung transplantation when using cytotoxic drugs [23, 33, 34]. Altogether, diseases belonging to the telomere syndrome may be considered multifactorial, resulting from strong genetic predisposition and toxic exposure such as smoking in case of ILD.

In the current study, transplant-free survival was lower with than without TERT/TERC DAV on both univariate and multivariate analysis. Telomeres length had been previously been associated with reduced transplant survival in the context of IPF [35]. Male sex, lung function test results, red blood cell macrocytosis, and thrombocytopenia were also associated with transplant-free survival. Assessing the survival of patients in the presence of competing events (transplantation) is tricky. The traditional approach (Kaplan-Meier estimator) assumes that the distribution of survival times of patients who underwent transplantation is the same as that of patients who did not (uninformative censoring). This assumption is obviously untenable here, and this approach overestimates the "true" survival of patients because the sickest patients are removed from the database over time. Therefore, we accounted for time to death or transplantation as an end-point and reported transplant-free survival.

The survival in the whole cohort seemed unexpectedly high (median survival 7.3 years) compared with that for sporadic IPF, for instance, which clearly indicates that the population suspected of TERT/TERC DAV is a subpopulation of those with ILD. In the largest series of patients with pulmonary fibrosis associated with TERT mutation reported to date, the mean survival was 3 years after diagnosis [9]. However, very long survival has been reported in patients with TERT mutation (27 years in one report) [36]. Moreover, more than half of the patients in the current series did not present IPF but rather other pulmonary diagnoses such as alveolar proteinosis or hypersensitivity pneumonitis, with less severe prognosis [37]. Patients may have been referred earlier because of familial history of pulmonary fibrosis, which may have resulted in increased survival. Age was not significantly associated with decreased transplant-free survival $(p=0.11)$. However, relatively younger patients were frequently referred for lung transplantation, which resulted in decreased transplant free-survival in this group.

Our study contains several limitations. Because of the retrospective nature of the study, some data are missing and a centre bias may exist; however, we did not find any centre effect for the prevalence of mutation or survival. Second, we did not confirm the impact of the mutations at a protein level with functional analysis or specific transfection, although telomere length and analysis of telomerase activity may be difficult to interpret $[38,39]$. However, we considered all the rare variants as possibly pathogenic mutations, as is usual in a genetic diagnostic process and actualised recommendations, although identification of causal variants is a challenge of current genetics $[18,19,40]$. Several mutations had been previously reported with or without functional analysis or specific transfection. Pathogenic scores (CADD score and polyphen) were predicted to be deleterious, although telomere lengths were not reduced in Southern blot analysis for all patients. Four other genes have been reported to cause telomere-mediated pulmonary fibrosis: RTEL1, PARN, DKC1 and TINF2 [30, 31, 41-43]. Indeed, the transplant-free survival in the group without TERT/TERC DAV could be affected by a mutation within one of these genes [44]. 
In conclusion, this series suggests that patients with ILD, idiopathic or non-idiopathic, who feature FPF or have extra-pulmonary signs suggestive of telomere syndrome, should undergo genetic testing. However, increasing the number of patients and genes analysed without conclusive functional analysis will also lead to increase in the number of variants of unknown significance, as reported for some patients of this cohort.

\section{Acknowledgements}

Collaborators: E. Magois, B. Toublanc (CHU d'Amiens, Amiens), A. Plessier (Hopital Beaujon, Clichy), A. Gondouin (Hopital Minjoz, Besancon), L. Savale (Hopital du Kremlin-Bicetre, Bicetre), D. Bouvry, B. Duchemann, A. Hervé, Y. Uzunhan, M. Zysman (Hopital Avicenne, Bobigny), B. Gentil (Hopital Oudot, Bourgoin), P. Bonniaud, L. Olivier-Faivre (CHU de Dijon, Dijon), A. Mehdaoui (Hopital Eure Seine, Evreux), J. Le Pavec (Centre chirurgical Marie Lannelongue, Le Plessis Robinson), N. Pottier, B. Wallaert (CHRU de Lille, Lille), C. Khouatra, L. Kiakouama, J. Traclet, P-J. Souquet (Hopitaux Pradel et Lyon sud, Lyon), C. Gomez (Hopital Nord, Marseille), A. Bourdin, V. Moulaire (CHRU de Montpellier, Montpellier), M. Aubier, O. Brugière, G. Dauriat, P. Dieudé, A. Marceau, M. Neuville, P. Pradère, C. Taillé (Hopital Bichat, Paris), L. Texeira (Hopital Cochin, Paris), S. Feuillet, L. Galicier, G. Lorillon, E. Oksenhendler, R.P. de la Tour, F. Riviere, F.S. de Fontbrune, Y. Tandjaoui (Hopital Saint Louis, Paris), M. Wislez, A. Olaiwan, H. Lioté (Hopital Tenon, Paris), J.F. Boitiaux (Hopital Dubos, Pontoise), P. Delaval (Hopital Pontchaillou, Rennes), S. Dominique (Hopital Nicolle, Rouen), S. Hirschi (Hôpitaux Universitaires de Strasbourg, Strasbourg), A. Didier, S. Ollier (Hopital Larrey, Toulouse), E. Manali, S. Papiris (Attikon University Hospital, Athens, Greece), S. Amselem, N. Nathan, A. Clement (Hopital Trousseau, Paris; all France).

Author contributions are as follows. Conception and design: R. Borie, L. Tabèze, G. Thabut, C. Kannengiesser, B. Crestani; analysis and interpretation: R. Borie, L. Tabèze, H. Nunes, V. Cottin, S. Marchand-Adam, G. Prevot, A. Tazi, J. Cadranel, H. Mal, L. Wemeau-Stervinou, G. Thabut, A. Bergeron Lafaurie, D. Israel-Biet, C. Picard, M. Reynaud Gaubert, S. Jouneau, J-M. Naccache, J. Mankikian, C. Ménard, P. Revy, J-F. Cordier, D. Valeyre, B. Grandchamp, C. Kannengiesser, B. Crestani; drafting the manuscript for important intellectual content: R. Borie, L. Tabèze, H. Nunes, V. Cottin, S. Marchand-Adam, J. Cadranel, L. Wemeau-Stervinou, G. Thabut, J-M. Naccache, J-F. Cordier, D. Valeyre, C. Kannengiesser, B. Crestani.

\section{References}

1 Hodgson U, Laitinen T, Tukiainen P. Nationwide prevalence of sporadic and familial idiopathic pulmonary fibrosis: evidence of founder effect among multiplex families in Finland. Thorax 2002; 57: 338-342.

2 Kropski JA, Pritchett JM, Zoz DF, et al. Extensive phenotyping of individuals at-risk for familial interstitial pneumonia reveals clues to the pathogenesis of interstitial lung disease. Am J Respir Crit Care Med 2014; 191: 417-426.

3 Tsakiri KD, Cronkhite JT, Kuan PJ, et al. Adult-onset pulmonary fibrosis caused by mutations in telomerase. Proc Natl Acad Sci USA 2007; 104: 7552-7557.

4 Armanios MY, Chen JJ, Cogan JD, et al. Telomerase mutations in families with idiopathic pulmonary fibrosis. N Engl J Med 2007; 356: 1317-1326.

5 Borie R, Crestani B, Dieude $\mathrm{P}$, et al. The MUC5B variant is associated with idiopathic pulmonary fibrosis but not with systemic sclerosis interstitial lung disease in the European Caucasian population. PLoS One 2013; 8: e70621.

6 6Armanios M. Telomeres and age-related disease: how telomere biology informs clinical paradigms. J Clin Invest 2013; 123: 996-1002.

7 Calado RT, Regal JA, Kleiner DE, et al. A spectrum of severe familial liver disorders associate with telomerase mutations. PLoS One 2009; 4: e7926.

8 Collopy LC, Walne AJ, Cardoso S, et al. Triallelic and epigenetic-like inheritance in human disorders of telomerase. Blood 2015; 126: 176-184.

9 Diaz de Leon A, Cronkhite JT, Katzenstein AL, et al. Telomere lengths, pulmonary fibrosis and telomerase (TERT) mutations. PLoS One 2010; 5: e10680.

10 Horimasu Y, Ohshimo S, Bonella F, et al. MUC5B promoter polymorphism in Japanese patients with idiopathic pulmonary fibrosis. Respirology 2015; 20: 439-444.

11 Seibold MA, Wise AL, Speer MC, et al. A common MUC5B promoter polymorphism and pulmonary fibrosis. N Engl J Med 2011; 364: 1503-1512.

12 Raghu G, Collard HR, Egan JJ, et al. An official ATS/ERS/JRS/ALAT statement: idiopathic pulmonary fibrosis: evidence-based guidelines for diagnosis and management. Am J Respir Crit Care Med 2011; 183: 788-824.

13 Travis WD, Costabel U, Hansell DM, et al. An official American Thoracic Society/European Respiratory Society statement: Update of the international multidisciplinary classification of the idiopathic interstitial pneumonias. Am J Respir Crit Care Med 2013; 188: 733-748.

14 Cottin V, Crestani B, Valeyre D, et al. Diagnosis and management of idiopathic pulmonary fibrosis: French practical guidelines. Eur Respir Rev 2014; 23: 193-214.

15 Savage SA, Bertuch AA. The genetics and clinical manifestations of telomere biology disorders. Genet Med 2010; 12: $753-764$.

16 World Health Organisation nutritional anaemias report of a WHO scientific group. World Health Organisation, Geneva, 1968

17 Raghu G, Collard HR, Egan JJ, et al. An official ATS/ERS/JRS/ALAT statement: idiopathic pulmonary fibrosis: evidence-based guidelines for diagnosis and management. Am J Respir Crit Care Med 2011; 183: 788-824.

18 Richards S, Aziz N, Bale S, et al. Standards and guidelines for the interpretation of sequence variants: a joint consensus recommendation of the American College of Medical Genetics and Genomics and the Association for Molecular Pathology. Genet Med 2015; 17: 405-424.

19 Claustres M, Kozich V, Dequeker E, et al. Recommendations for reporting results of diagnostic genetic testing (biochemical, cytogenetic and molecular genetic). Eur J Hum Genet 2014; 22: 160-170.

20 Therneau TM, Grambsch PM. Modeling survival data: extending the Cox model. Springer, New York, 2000. 
21 Marchand-Adam S, Diot B, Magro P, et al. Pulmonary alveolar proteinosis revealing a telomerase disease. Am J Respir Crit Care Med 2013; 188: 402-404.

22 Nunes H, Monnet I, Kannengiesser C, et al. Is telomeropathy the explanation for combined pulmonary fibrosis and emphysema syndrome?: report of a family with TERT mutation. Am J Respir Crit Care Med 2014; 189: 753-754.

23 Borie R, Kannengiesser C, Hirschi S, et al. Severe hematologic complications after lung transplantation in patients with telomerase complex mutations. J Heart Lung Transplant 2015; 34: 538-546.

24 Cottin V, Crestani B, Valeyre D, et al. Recommandations pratiques pour le diagnostic et la prise en charge de la fibrose pulmonaire idiopathique. Elaborees par le centre national de reference et les centres de competence pour les maladies pulmonaires rares sous l'egide de la Societe de pneumologie de langue francaise. [French practical guidelines for the diagnosis and management of idiopathic pulmonary fibrosis. From the National Reference and the Competence centers for rare diseases and the Societe de Pneumologie de Langue Francaise.] Rev Mal Respir 2013; 30: 879-902.

25 Diaz de Leon A, Cronkhite JT, Yilmaz C, et al. Subclinical lung disease, macrocytosis, and premature graying in kindreds with telomerase (TERT) mutations. Chest 2011; 140: 753-763.

26 Vulliamy T, Marrone A, Dokal I, et al. Association between aplastic anaemia and mutations in telomerase RNA. Lancet 2002; 359: 2168-2170.

27 Parry EM, Alder JK, Qi X, et al. Syndrome complex of bone marrow failure and pulmonary fibrosis predicts germline defects in telomerase. Blood 2011; 117: 5607-5611.

28 Gorgy AI, Jonassaint NL, Stanley SE, et al. Hepatopulmonary syndrome is a frequent cause of dyspnea in the short telomere disorders. Chest 2015; 148: 1019-1026.

29 Okamoto T, Miyazaki Y, Tomita M, et al. A familial history of pulmonary fibrosis in patients with chronic hypersensitivity pneumonitis. Respiration 2013; 85: 384-390.

30 Stuart BD, Choi J, Zaidi S, et al. Exome sequencing links mutations in PARN and RTEL1 with familial pulmonary fibrosis and telomere shortening. Nat Genet 2015; 47: 512-517.

31 Kannengiesser C, Borie R, Menard C, et al. Heterozygous RTEL1 mutations are associated with familial pulmonary fibrosis. Eur Respir J 2015; 46: 474-485.

32 Calado RT, Brudno J, Mehta P, et al. Constitutional telomerase mutations are genetic risk factors for cirrhosis Hepatology 2011; 53: 1600-1607.

33 Silhan LL, Shah PD, Chambers DC, et al. Lung transplantation in telomerase mutation carriers with pulmonary fibrosis. Eur Respir J 2014; 44: 178-187.

34 Tokman S, Singer JP, Devine MS, et al. Clinical outcomes of lung transplant recipients with telomerase mutations. J Heart Lung Transplant 2015; 34: 1318-1324.

35 Stuart BD, Lee JS, Kozlitina J, et al. Effect of telomere length on survival in patients with idiopathic pulmonary fibrosis: an observational cohort study with independent validation. Lancet Respir Med 2014; 2: 557-565.

36 El-Chemaly S, Ziegler SG, Calado RT, et al. Natural history of pulmonary fibrosis in two subjects with the same telomerase mutation. Chest 2011; 139: 1203-1209.

37 Flaherty KR, Thwaite EL, Kazerooni EA, et al. Radiological versus histological diagnosis in UIP and NSIP: survival implications. Thorax 2003; 58: 143-148.

38 Stanley SE, Chen JJ, Podlevsky JD, et al. Telomerase mutations in smokers with severe emphysema. J Clin Invest 2015; 125: 563-570.

39 Marrone A, Sokhal P, Walne A, et al. Functional characterization of novel telomerase RNA (TERC) mutations in patients with diverse clinical and pathological presentations. Haematologica 2007; 92: 1013-1020.

40 MacArthur DG, Manolio TA, Dimmock DP, et al. Guidelines for investigating causality of sequence variants in human disease. Nature 2014; 508: 469-476.

41 Cogan JD, Kropski JA, Zhao M, et al. Rare variants in RTEL1 are associated with familial interstitial pneumonia. Am J Respir Crit Care Med 2015; 191: 646-655.

42 Alder JK, Stanley SE, Wagner CL, et al. Exome Sequencing Identifies Mutant TINF2 in a Family With Pulmonary Fibrosis. Chest 2015; 147: 1361-1368.

43 Alder JK, Parry EM, Yegnasubramanian S, et al. Telomere phenotypes in females with heterozygous mutations in the dyskeratosis congenita 1 (DKC1) gene. Hum Mutat 2013; 34: 1481-1485.

44 Kropski JA, Blackwell TS, Loyd JE. The genetic basis of idiopathic pulmonary fibrosis. Eur Respir J 2015; 45: $1717-1727$. 